\title{
Postembryonic Neurogenesis in the CNS of the Tobacco Hornworm, Manduca sexta. II. Hormonal Control of Imaginal Nest Cell Degeneration and Differentiation During Metamorphosis
}

\author{
Ronald Booker and James W. Truman \\ Department of Zoology, University of Washington, Seattle, Washington 98195
}

The nervous system of an adult moth is comprised of retained larval neurons that are remodeled during metamorphosis and a set of new adult specific neurons. The new neurons arise from a stereotyped array of stem cells (neuroblasts) that divide during larval life to generate nests of up to 100 arrested postmitotic immature neurons, the imaginal nest (IN) cells. At the onset of metamorphosis, some of the IN cells die while the remainder differentiate into mature functional neurons.

Metamorphosis in insects is regulated by 2 classes of hormones, the ecdysteroids and the juvenile hormones. The transition from larva to pupa requires the disappearance of juvenile hormones followed by 2 releases of ecdysteroids: a small "commitment peak" and a larger "prepupal peak." Through a series of endocrine manipulations, we demonstrate that the death and differentiation observed among the abdominal IN cells at metamorphosis are both influenced by these hormonal cues. If the abdomen was isolated from the hormonal sources in the anterior half of the larva before the onset of metamorphosis, death and differentiation of the IN cells were prevented. Infusion of ecdysteroids into such abdomens, to mimic the prepupal peak, resulted in the IN cells showing the same fate as seen in control animals during the early phases of metamorphosis.

The response of the IN cells to the small commitment peak of ecdysteroids was heterogeneous. Exposure to this small peak of steroids caused some cells to become committed to resume their development, making them resistant to juvenile hormone application. For other cells within the same nest, final differentiation could still be blocked by juvenile hormones even after exposure to the commitment pulse. The proportion of cells within a nest that became committed by the small peak of ecdysteroids was lineage dependent.

In the moth Manduca sexta, the neurons that make up the adult CNS are derived from 2 sources: larval neurons, some of which are remodeled to serve new functions in the adult (Taylor and Truman, 1974; Truman and Reiss, 1976; Levine and Truman, 1982,1985 ), and adult specific neurons, which are added to the

\footnotetext{
Received Feb. 18, 1987; revised May 28, 1987; accepted June 3, 1987.

We thank Dr. Shirley Reiss for technical assistance during some phases of this study, and Drs. Susan Fahrbach, David Morton, Jane Witten, and Ms. Cathy Fittinghoff for a critical reading of the manuscript. This work was supported by grants from NIH (ROI-NS-13079) and the McKnight Foundation to J.W.T. and a NIH postdoctoral fellowship (F32 NS070160) to R.B.

Correspondence should be addressed to Dr. Ronald Booker, Department of Zoology, Kincaid Hall NJ-15, University of Washington, Seattle, WA 98195

Copyright (C) 1987 Society for Neuroscience $0270-6474 / 87 / 124107-08 \$ 02.00 / 0$
}

CNS during metamorphosis (Booker and Truman, 1987). The latter cells are the progeny of identifiable stem cells (neuroblasts), which are found in all of the segmental ganglia. By the end of larval life, the neuroblasts, through a series of asymmetrical divisions, generate nests of up to 100 postmitotic predifferentiative neurons, the imaginal nest (IN) cells. At the onset of metamorphosis, some of these cells die, while the remainder differentiate into mature neurons. They contribute 2500-3000 new neurons to each thoracic ganglion of the adult. In the simpler abdominal ganglia only about 50 cells are added.

Postembryonic development in insects is under the control of 2 classes of hormones: the ccdystcroids and the antimetamorphic juvenile hormones (JH) (Riddiford, 1980). Metamorphosis is preceded by a fall in the titers of JH in the middle of the 5 th (final) larval instar. This fall of JH is followed by a small rise in the level of ecdysteroids, the "commitment peak," which in epidermal and other larval tissues switches the developmental program of the cells from a larval to a pupal program. A second and larger peak of ecdysteroids then triggers the onset of pupal differentiation. $\mathrm{JH}$ reappears transiently during this second ecdysteroid peak (the prepupal peak) to prevent precocious adult development of certain imaginal disc structures (Kiguchi and Riddiford, 1978).

Previous reports have shown that the ecdysteroids and JHs have an important role in regulating the death or remodeling of functional larval neurons at metamorphosis (Truman and Schwartz, 1984; Bennett and Truman, 1985; Weeks and Truman, 1985, 1986; Levine et al., 1986). Here we report that the maturation or death of the immature IN cells that occurs during metamorphosis is also influenced by these 2 classes of hormones.

\section{Materials and Methods}

Experimental animals. Larvae of the tobacco hornworm $M$. sexta were reared on an artificial diet under conditions of $26^{\circ} \mathrm{C}$ and a $17 \mathrm{~L}: 7 \mathrm{D}$ (lightson 7 AM; lights-off 12 AM) photoperiod as described by Bell and Joachim (1978). Under these rearing conditions, approximately $18 \mathrm{~d}$ were required from hatching to pupal ecdysis and $20 \mathrm{~d}$ from pupal ecdysis to the emergence of the adult. The ecdyses of the various stages, the 4th, 5 th, and pupal ecdyses were used as references in staging the insects $(4-0,5-0$, and $P-0$ respectively). Late in the last (5th) larval instar the caterpillar ceases feeding and begins to search for an appropriate place to pupate. The start of this "wandering" behavior marks the onset of metamorphosis and was also used for staging ( $\mathrm{W}-0$ ).

Histological techniques. The ganglia were removed from $\mathrm{CO}_{2}$-anesthetized animals and prepared for histological analysis as follows. For paraffin sectioning, the ganglia were fixed overnight in alcoholic or aqueous Bouin's fixative at room temperature, dehydrated through a graded ethanol series, and embedded. Ganglia were serially sectioned at $10 \mu \mathrm{m}$, and the sections stained with hematoxylin and eosin. For 
plastic sectioning, ganglia were fixed in a mixture of $2 \%$ glutaraldehyde and $4 \%$ paraformaldehyde (in phosphate buffer, $\mathrm{pH} 7.5$ ) overnight at $4^{\circ} \mathrm{C}$. The tissue was then dehydrated in a methanol series and embedded in Epon. The ganglia were cut serially at 2-5 $\mu \mathrm{m}$ and the sections stained with Richardson's stain (Richardson et al., 1960).

The birth dates of cells within the CNS were determined by allowing larvae to feed on a diet containing ${ }^{3} \mathrm{H}$-thymidine $(50 \mu \mathrm{Ci} / \mathrm{ml}$ diet $)$. The distribution of the label was visualized using standard autoradiographic techniques (Booker and Truman, 1987).

Estimates of the number of cells within the nests were based on counts of cellular profiles. Cell counts based on sectioned material overestimate the number of cells present because a given cell may appear in more than one section. We corrected our raw counts using the method of Abercrombie (1946) based on the equation $N=n(t / t+d)$, where $N$ equals the estimated number of cells, $n$ is the number of profiles counted in the sections, $t$ is the thickness of the section, and $d$ is the average diameter of the cells being counted. The number of degenerating cells within a nest was estimated by counting the number of densely staining pycnotic bodies, which were assumed to be the remains of degenerating IN cells.

Endocrine manipulations. The sole source of ecdysone in Manduca larvae are the prothoracic glands located in the first thoracic segment, while JHs are released from the corpora allata located in the head. Consequently, the placement of a ligature between the thorax and the abdomen prevents the latter from receiving the normal surges of both the ecdysteroids and JHs. Anesthetized larvae were ligated between the thorax and the abdomen using silk suture thread (size 00). The anterior part of the body was cut off and the wound treated with phenylthiourea to prevent melanization of the hemolymph. All larvae were ligated between 9:00 and 11:00 AM.

Ecdysteroids were administered to isolated abdomens by infusion of 20-hydroxyecdysone (20-HE; Sigma Chemical Co., St. Louis; or Rohto Pharmaceutical Co. Ltd., Osaka, Japan). Twenty-HE was dissolved in saline (Ephrussi and Beadle, 1936) and adjusted to a concentration of approximately $1 \mathrm{mg} / \mathrm{ml}$ using ultraviolet spectrophotometry (Meltzer, 1971). The ligated abdomens were anesthetized in $\mathrm{CO}_{2}$, the tip of the dorsal horn was removed, and polyethylene tubing (PE-10; Intermedic, Parsippany, NJ) was inserted through the opening into the hemolymph. The tubing was held in place with silk thread $(000)$ and then anchored with melted wax (CSC Softseal Tackiwax, Central Scientific Co., Chicago). Typically, infusion of $20-\mathrm{HE}$ was carricd out at a constant rate of $5.4 \mu \mathrm{l} / \mathrm{hr}$ for a period of $12 \mathrm{hr}$ using $100 \mu \mathrm{l}$ syringes (Unimetrics Co., Anaheim, CA) driven by a variable-speed syringe pump (Sage Instruments, Cambridge, MA). At the end of the infusion the tubing was fused close to the dorsal horn using hot forceps.

In order to investigate the role of the JHs in the metamorphosis of the IN cells, we used the JH mimic (JHM), methoprene (Zoecon Corp., Palo Alto, CA). The methoprene was dissolved in cyclohexane at a concentration of $1 \mathrm{mg} / \mathrm{ml}$. On the day of ligation, $10 \mu \mathrm{g}$ of the analog was applied topically to the isolated abdomens. When this dose was given to intact larvae prior to the "commitment peak," it delayed the onset of wandering by 2-3 d. The following day some of the JHMtreated abdomens were infused with $20-\mathrm{HE}$ as described above.

\section{Results}

Time course of events during postembryonic neurogenesis

Neuroblasts are present in all the segmental ganglia of larval Manduca (Booker and Truman, 1987), the number varying in a segment-specific pattern. The thoracic ganglia have 45-47 neuroblasts, whereas most of the abdominal ganglia (A3-A7) possess only 8 ( 4 pairs). The neuroblasts begin mitosis during the late 2 nd or early 3 rd larval instar. They divide asymmetrically, producing a second neuroblast and a small ganglion mother cell. The ganglion mother cells divide once symmetrically, generating 2 IN cells. By late in the 5th and final larval instar, each neuroblast is associated with a nest of 10-100 IN cells (Fig. $1 B$ ). All of these progeny are small cells $(\sim 5 \mu \mathrm{m})$. They possess only a thin rim of cytoplasm, have no distinct nucleolus, and send an axonlike process into the neuropil. Regardless of when they were born during larval life, they arrest their development at this immature stage. Therefore, although their birth dates may differ by as much as $10 \mathrm{~d}$, the morphologies of the oldest and the youngest IN cells in a larval nest are identical.

Coincident with the onset of wandering behavior, the arrested IN cells resume their development. Depending on their segmental location and neuroblast of origin, some of the IN cells in a nest die (Fig. 1C), while the remainder differentiate into adult-specific neurons (Fig. $1 D$ ). Little cell death is observed in the thoracic nests, whereas it is extensive in most of the abdominal nests. As the surviving IN cells mature into adult neurons, their somata enlarge due to an increase in both nuclear and cytoplasmic volume, and their nucleoli become more pronounced. Depending upon its identity, an abdominal neuroblast may contribute up to 20 new neurons to the adult CNS, while the thoracic neuroblasts contribute up to 100 or more cells. Most of the mature cells are small (10-15 $\mu \mathrm{m}$ in diameter). In the thoracic and fused terminal ganglia, there are a few cells that measure $30-40 \mu \mathrm{m}$ in diameter.

\section{Effects of ligation on IN cell death and growth}

The resumption of IN cell development is temporally correlated with the hormonal events that bring about the larval-pupal transition (Fig. 1A). Both degeneration and growth of the IN cells occur after the larvae experience the small commitment peak of ecdysteroids, which occurs in the absence of the JHs. In order to determine if these endocrine events influence the fate of the IN cell, we isolated abdomens at various times during the larval-pupal transition, thereby preventing the abdominal CNS from being exposed to the subsequent hormonal pulses from the anterior end. The fates of the IN cells were determined $6 \mathrm{~d}$ later. The developmental fate of the IN cells between nests is qualitatively similar, with each nest differing only in the extent of cell loss and the final morphology of the mature neurons. Thus, the effect of the various manipulations that were performed were ascertained by following the fate of 2 of the 4 nests in the fourth abdominal ganglion [M and N-T(A4)].

The effect of ligation was strongly dependent upon the time that the manipulation was performed. In the M(A4) nest, isolation of the abdomens up until the first day of the wandering period prevented cell death (Fig. 2). Indeed, in abdomens isolated on the 2 nd or 3 rd day of the 5 th instar, the number of cells found in the M(A4) nest continued to increase after ligation. This increase was presumably due to the continued activity of the $\mathrm{M}$ neuroblast following ligation. During the first $48 \mathrm{hr}$ of wandering ( $W-0, W-1)$, abdomen isolation resulted in a stablization of the number of cells within the nest; the number of cells counted $6 \mathrm{~d}$ after isolation remained the same as at the time of ligation. When a ligature was applied on or after the 2nd day of wandering ( $W-2)$, after the caterpillars had experienced about half of the large prepupal peak of ecdysteroids, the number of IN cells counted in the nest dropped significantly. However, it did not drop to the 11-18 cells normally found in the M(A4) nest of adult moths. Even when abdominal ligatures were applied to pharate pupae that had experienced the complete prepupal peak of ecdysteroids, the number of cells in M(A4) did not then fall to the number found in controls 2-3 d after pupal ecdysis. Abdomens isolated at this point in development typically had 5-10 extra cells in their $M$ nests.

The N-T(A4) nest responded in a somewhat different fashion to the same ligation paradigm (Fig. 2). In control larvae, the 22 or so cells present in this nest at the onset of wandering had all disappeared $3 \mathrm{~d}$ after pupal ecdysis, with the first drop seen on 


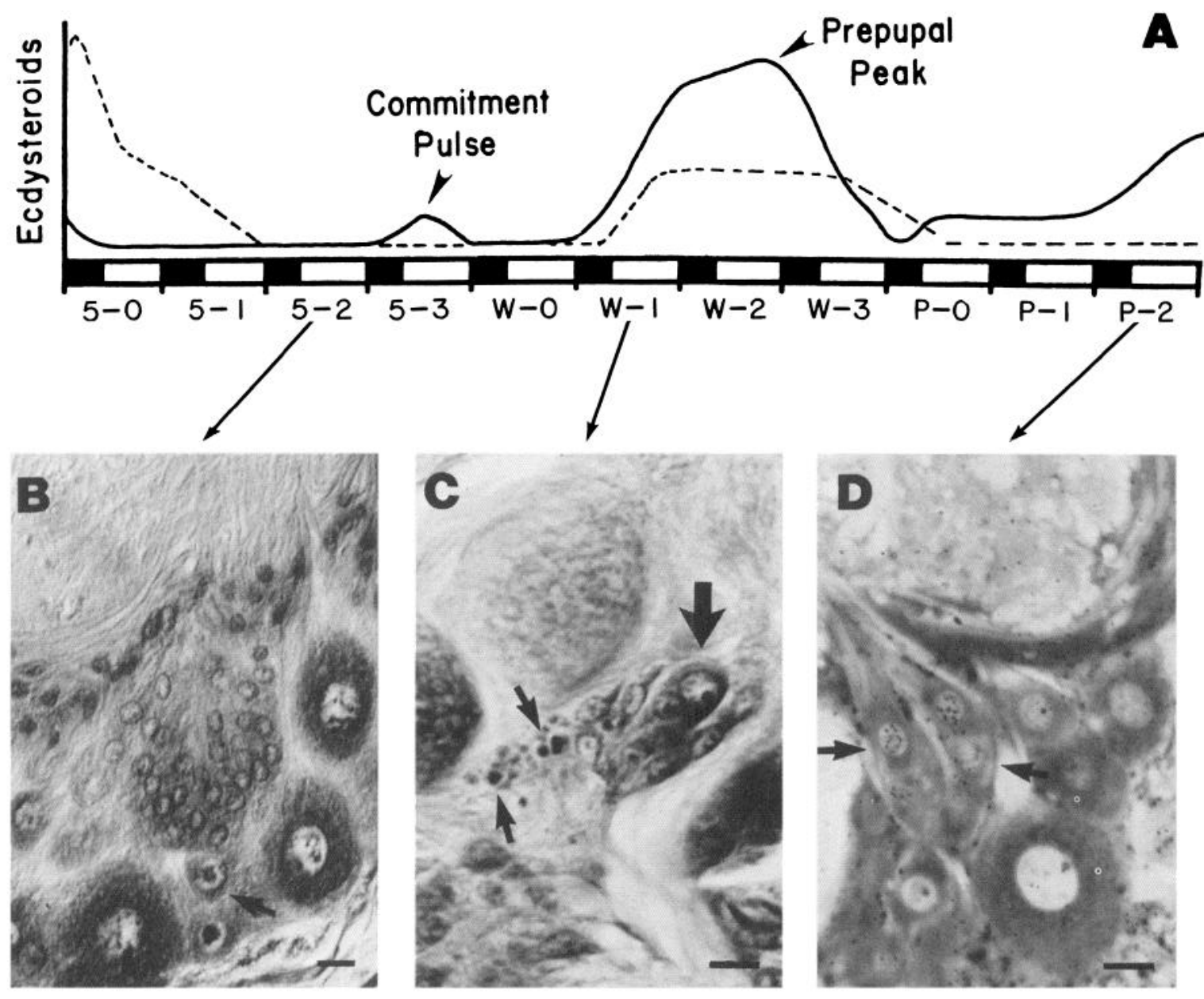

Figure 1. A, Hemolymph titers of juvenile hormones (dotted line) and ecdysteroids (solid line) around the time of the larval-pupal transition. The dark and light bars represent the photoperiod. The start of the 5th larval, wandering, and pupal stages are represented by $5-0$, W -0 , and $\mathrm{P}-0$, respectively. Photomicrographs of sections through nests in the fourth abdominal ganglion at various stages showing the state of the cells within the nests. $B, \mathrm{M}$ nest on the second day of the 5th instar showing the neuroblast (arrow) and its associated nest of arrested progeny. $C$, N-T nest on the first day of wandering showing the neuroblast (large arrow) pycnotic profiles (small arrows) in the N-T(A4) nest on the first day of wandering. $D$, Autoradiograph of a section through the $\mathrm{M}$ nest of a 2-d-old pupa that had been fed ${ }^{3} \mathrm{H}$-thymidine as a larva. Illustrating the growth of the IN cell somata (arrows) during during the early phases of metamorphosis. The presence of silver grains over the nucleolus confirms the larval origin of the cells. Scale bars, $10 \mu \mathrm{m}$. Hormone titers are based on Riddiford (1980).

the 2 nd day of wandering. In this nest ligation on the first day of wandering (W -1$)$ was already too late to prevent a fall in the number of cells in this nest. Ligation a day later $(\mathrm{W}-2)$ resulted in the survival of only about 5-7 cells in the N-T nest. Ligation on the next $2 \mathrm{~d}$ of the wandering stage gave the same result. As with the $M$ nest, the cell number did not drop to the levels seen in control animals $3 \mathrm{~d}$ after pupal ecdysis. Consequently, for both nests, there appear to be 2 discrete times during which anterior signals are required for IN cell degeneration. The first period extends from the 1st to the 3rd day of wandering, while the second period appears to occur after pupal ecdysis.

The effect of abdominal isolation on the growth of the IN cells was also ascertained. As mentioned above, in the case of the N-T nest, no cells normally persist to the adult stage. Accordingly, the surviving cells in the N-T nests never increased in size following ligation. Their morphology always remained larval-like.

Abdominal ligature on the 2 nd or 3rd day of the 5th larval instar prevented not only the degeneration of the IN cells in the M(A4) nest but also their growth. Five to $6 \mathrm{~d}$ after abdominal ligation, all the cells remained larval-like, measuring about 5 $\mu \mathrm{m}$ in diameter and possessing the thin rim of cytoplasm characteristic of premetamorphic larval cells (Fig. 3). The first indication of soma growth among the cells within this nest was seen in abdomens ligated at the onset of wandering ( $\mathrm{W}-0$ ). Ligation on successive days prior to pupal ecdysis resulted in a steady increase in both the number of growing IN cells and the extent of growth that they exhibited. By the day of pupation, ligation resulted in some cells continuing to grow to a size comparable to that seen $2-3$ d after pupation, whereas other cells in the nest showed no growth at all. In abdomens isolated on the 2 nd day of wandering and after, it was clear that the number of IN cells that enlarged was equivalent to the number expected to survive to the adult stage. It appears that by this stage there are 2 discrete populations of IN cells: one group that had begun the process of becoming mature imaginal neurons and a second that remained larval-like. Ignoring the latter group in ganglia from animals ligated on the day of pupation, it was still evident that ligation inhibited the postpupation growth normally shown by some of the neurons (Figs. 3, 5). Thus, anterior factors appear 


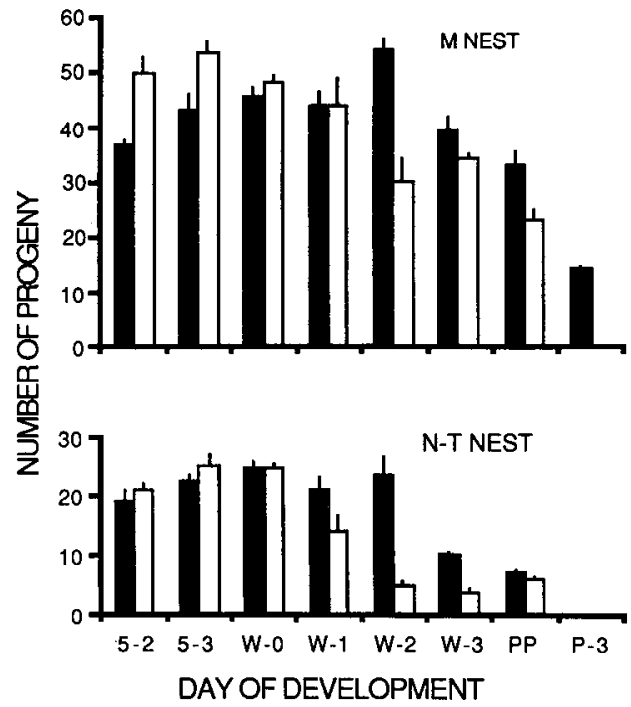

Figure 2. Effect of abdominal ligation on the number of IN cells in selected nests in ganglion A4. The abdomens were ligated on each of the days shown. Upper panel, cells in the M nest; lower panel, cells in the N-T nest. For each pair of histograms the filled bars represent the number of cells present at the time of ligation; the open histograms, the number of cells found in a nest $6 \mathrm{~d}$ after ligation. Each bar represents the mean \pm SEM corrected cell counts for an average of 8 nests. PP, pharate pupa.

to influence IN cell growth during the period prior to pupal ecdysis, starting from the onset of wandering, and also during the first 2-3 d after the pupal molt.

\section{Ecdysteroid infusions}

The ligation experiments suggest that, around the time of the onset of wandering, humoral signals from the anterior half of the larvae influence the fate of the IN cells. The period during which the most extensive rounds of cell death and growth occurred among the IN cells was temporally correlated with the release of the large prepupal peak of ecdysteroids responsible for the formation of the pupal stage. To determine if ecdysteroid exposure was necessary for these changes in the fate of the IN cells, we isolated abdomens from larvae that had initiated wandering behavior. On the following day, in order to mimic the prepupal peak of ecdysteroids, we infused a subset of the abdomens with 60-65 $\mu \mathrm{g}$ of 20-HE over $12 \mathrm{hr}$, a dosage sufficient to trigger pupal differentiation of the epidermis of isolated abdomens (Nijhout, 1976).

Ligation of abdomens on $\mathrm{W}-0$ prevented both the major waves of cell loss and growth that occur in the IN cells following the onset of wandering behavior (Figs. 2-5). The normal patterns of death and growth were restored within $24 \mathrm{hr}$ after the initiation of the 20-HE infusion. The pattern observed within individual nests was very similar to that seen in intact controls during the early phases of metamorphosis (Fig. 4). Specifically, in the $\mathrm{N}-\mathrm{T}(\mathrm{A} 4)$ nest the appearance of pycnotic cells $24 \mathrm{hr}$ after the initiation of the infusion was accompanied by a $30 \%$ drop in the number of cells counted. By the following day, the number of cells within this nest stabilized between $5-8$ cells. By contrast, in the $M$ nest there were only a few pycnotic cells present on the day following infusion; the peak in the numbers of dying cells was evident on the following day. Within $3 \mathrm{~d}$ after infusion, the number of cells in the $M$ nest was equivalent to that seen

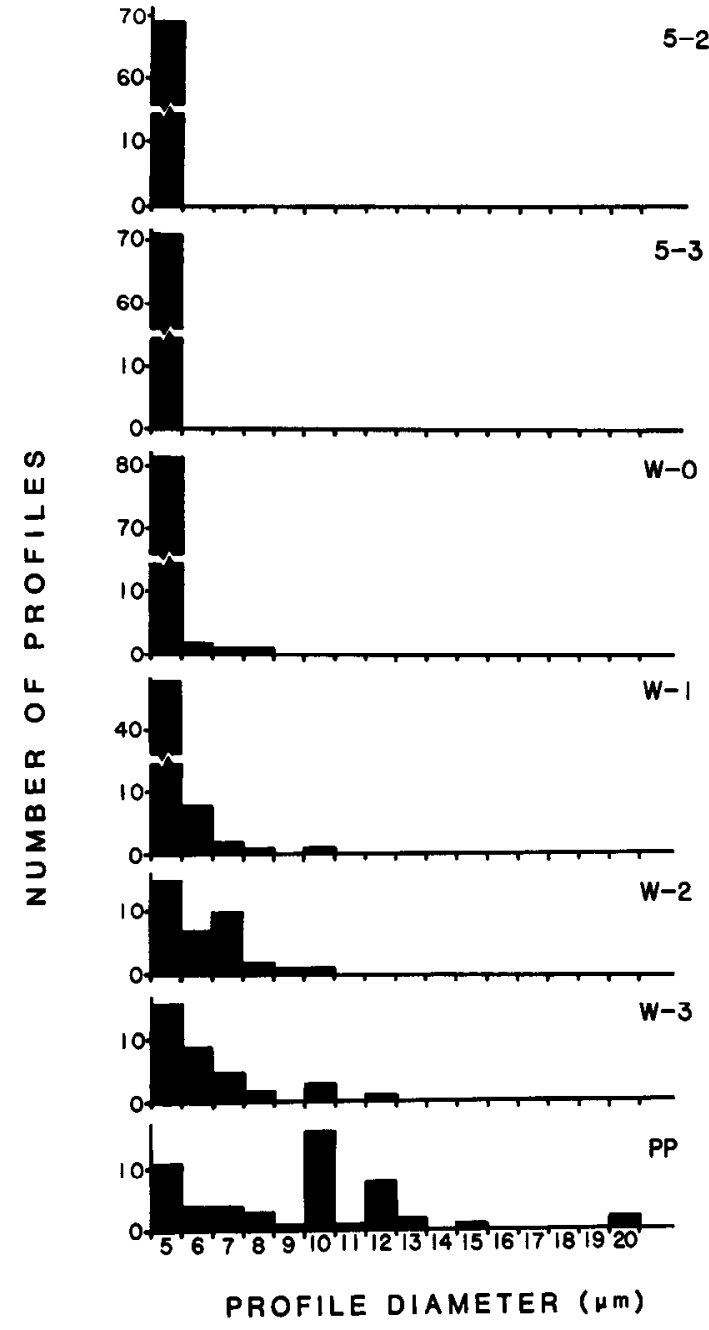

Figure 3. Size distribution of IN cell profiles in the $\mathrm{M}$ nest in abdomens isolated at various times during the larval-pupal transition. All abdomens were isolated on the days indicated and sacrificed $6 \mathrm{~d}$ later.

in intact animals on the day of pupal ecdysis, but it then stablized at a higher number than that subsequently seen in intact animals.

A predictable subset of the IN cells in A4 responded to the infusion of $20-\mathrm{HE}$ with an expansion of soma diameter. For example, in the $M$ nest, 11-18 cells began to enlarge noticeably by the day after infusion (Figs. $4 A$ and $5 B$ ). Over the next several days there was a steady increase in the average diameter of these cells, a plateau of $7 \mu \mathrm{m}$ being reached, $3 \mathrm{~d}$ after infusion. At no time did the number of enlarging cells ever exceed the number of cells found in the mature adult ganglion. The growth of the cells in the infused abdomens was retarded compared to that seen in intact controls. Three days after the pupal molt, controls showed an additional spurt of IN soma growth which brought their cell diameters to $11-12 \mu \mathrm{m}$, while in the infused abdomens no second growth spurt was seen.

\section{Role of the commitment peak in IN cell fate}

While the prepupal peak of ecdysteroids has a major role in triggering both IN cell death and growth, a few pycnotic cells and the enlargement of a few cells was evident around the time of the release of the small commitment peak of ecdysteroids, suggesting that this hormone pulse also plays a role in the fate 
A
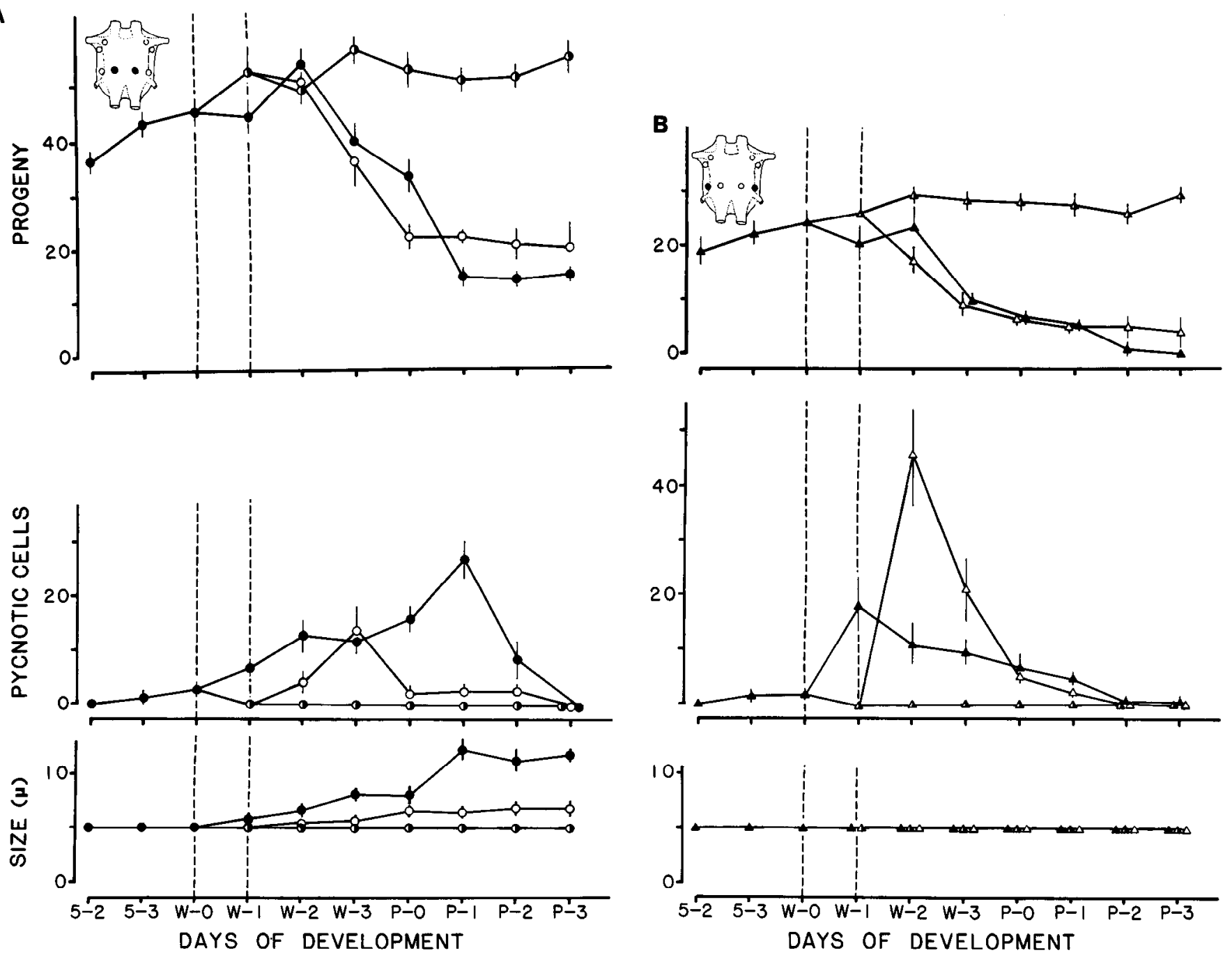

Figure 4. Effect of steroid manipulation in the fate of the IN cells. $A$, Corrected cell counts of the $\mathrm{M}$ and $B$, N-T nests, respectively, in A4. Filled symbols, intact controls; half-filled symhols, ligated abdomens; and open symbols, ligated and infused abdomens. All abdomens were ligated on W - O (first dashed line). The following day, a subset of these were infused with 20-HE (second dashed line). Animals from each group were sacrificed on the days indicated. Top panel, Corrected number of cells in each nest; middle panel, number of pycnotic cells; bottom panel, soma size of the IN cells. The points represent the means \pm SEM for an average of 8 nests.

of the IN cells. To better understand the influence of the commitment peak in the fate of the IN cells, we isolated abdomens prior to the commitment peak $(5-2)$. On the following day, we infused a subset of these abdomens with the same dosage of 20 -HE ( $\sim 60-65 \mu \mathrm{g}$ over $12 \mathrm{hr}$ ) as was given to abdomens iso- lated from wandering animals. This treatment triggered death of some IN cells and the growth of others, but it was less effective than when given to abdomens isolated after the commitment peak (Table 1). In both the M and N-T(A4) nests the extent of cell loss was $60-70 \%$ of that observed when the same amount

Table 1. Effect of various hormonal manipulations on the corrected cell counts in the $M$ and $N-T$ tests in $A 4$

\begin{tabular}{|c|c|c|c|c|}
\hline \multirow[b]{3}{*}{ Type of treatment } & \multicolumn{4}{|l|}{ Time of Ligation } \\
\hline & \multicolumn{2}{|l|}{$5-2$} & \multicolumn{2}{|l|}{$\mathbf{W}-\mathbf{O}$} \\
\hline & $\mathrm{M}$ nest $^{c}$ & N-T nestc & M nest ${ }^{c}$ & N-T nest ${ }^{c}$ \\
\hline Ligation & $49.5 \pm 2.7(n=6)$ & $20.8 \pm 1.0(n=6)$ & $50.8 \pm 1.3(n=6)$ & $25.9 \pm 1.1(n=6)$ \\
\hline Ligation $+\mathrm{JHM}^{a}$ & $48.5 \pm 2.4(n=8)$ & $26 \pm 0.7(n=8)$ & $48.7 \pm 1.5(n=14)$ & $25.3 \pm 1.0(n=14)$ \\
\hline Ligation $+\mathrm{JHM}^{a}+20-\mathrm{HE}^{b}$ & $46.5 \pm 2.3(n=10)$ & $22.2 \pm 1.3(n=10)$ & $48.2 \pm 2.0(n=18)$ & $15.4 \pm 1.7(n=18)$ \\
\hline Ligation $+20-\mathrm{HE}^{b}$ & $31.2 \pm 2.8(n=6)$ & $10.8 \pm 1.9(n=6)$ & $20.2 \pm 2.0(n=8)$ & $5.0 \pm 1.2(n=8)$ \\
\hline
\end{tabular}

Larvae were ligated either before $(5-2)$ or after $(\mathrm{W}-\mathrm{O})$ the small commitment pulse of ecdysteroids.

${ }^{a} 10 \mu \mathrm{g}$ applied topically.

${ }^{\circ} 60-65 \mu \mathrm{g}$ infused over $12 \mathrm{hr}$.

c Number of cells present in each nest $6 \mathrm{~d}$ after ligation; mean \pm SEM. 
Figure 5. Photomicrographs illustrating the effect of hormonal manipulations on the morphology of IN cells. $A$, Section through the M(A4) nests $7 \mathrm{~d}$ after ligation on $\mathrm{W}-0$. The morphology of the cells in the nest remains larval-like, there being no signs of further IN cell development. $B$, Photomicrograph of $\mathrm{M}(\mathrm{A} 4)$ of an isolated abdomen $5 \mathrm{~d}$ after infusion of 20 -HE. The infusion not only triggered cell death, as can be seen by the presence of pycnotic profiles (small arrow), but there was a substantial increase in the diameter of some of the surviving IN cells (large arrow). Scale bars, $10 \mu \mathrm{m}$.
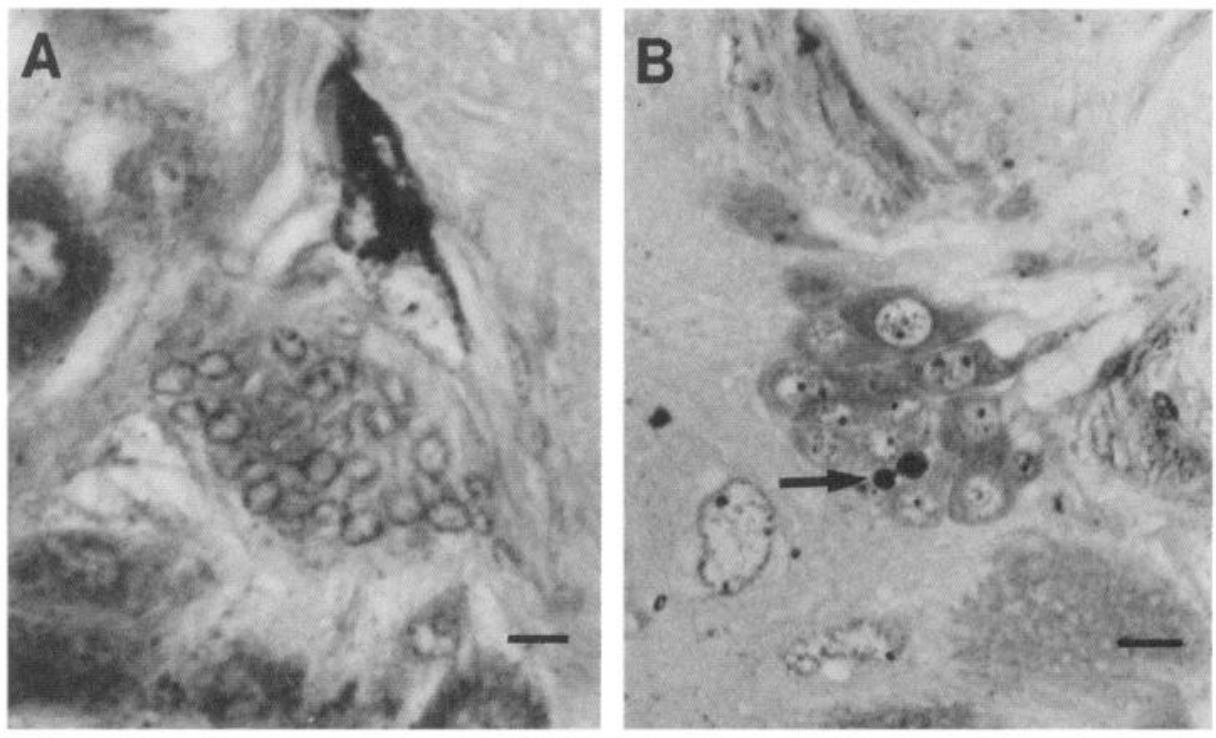

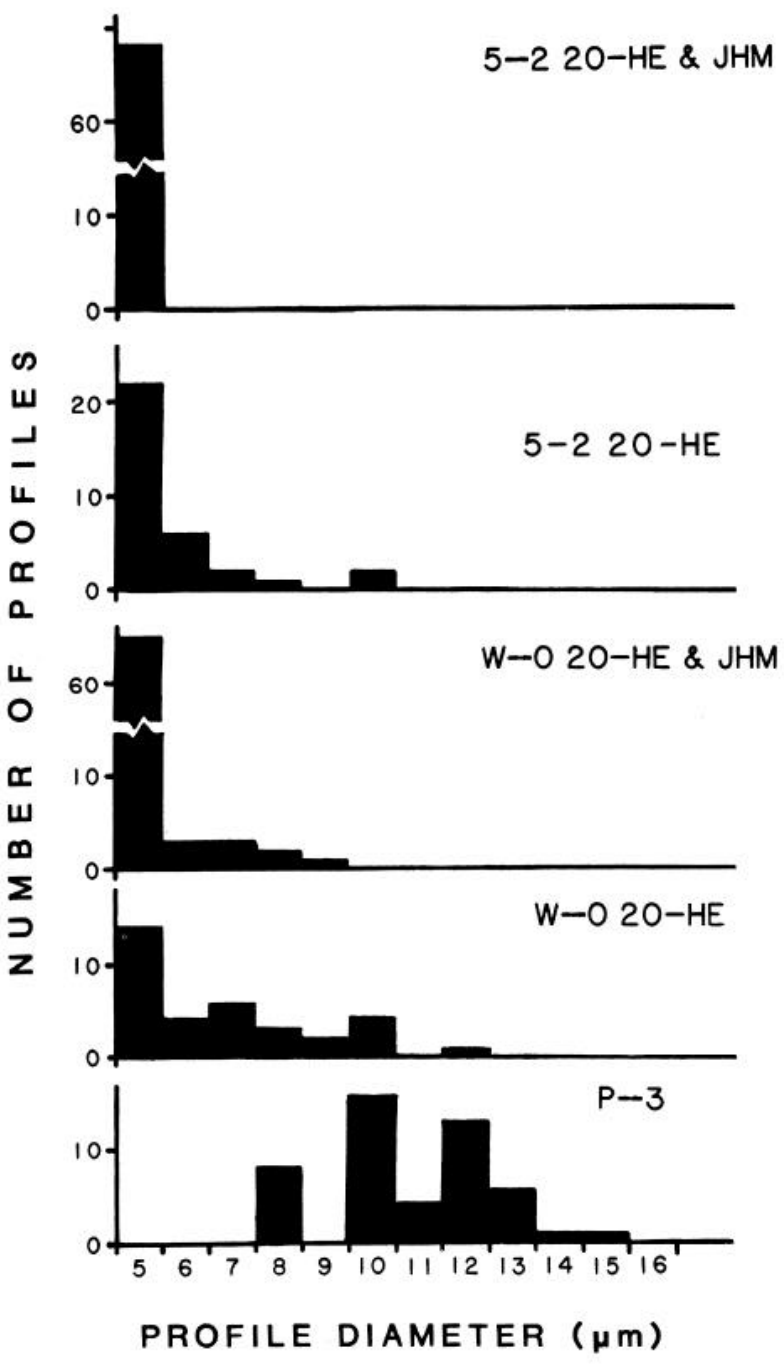

Figure 6. Size distribution of IN cell profiles in the $\mathrm{M}$ nest in abdomens isolated before $(5-2)$ and after wandering $(\mathrm{W}-0)$ and subsequently treated with $20-\mathrm{HE}$ alone $(60-65 \mu \mathrm{g}$ over $12 \mathrm{hr}$ ) or with both 20 -HE and JHM (10 $\mu \mathrm{g}$ applied topically). The size distribution of cells in a nest from an intact animal sacrificed $3 \mathrm{~d}$ after pupation is included for comparison. of 20-HE was infused into abdomens isolated from wandering larvae. Also, fewer cells in the M(A4) nest in abdomens isolated prior to wandering responded to the infusion of 20 -HE with growth. In these abdomens only 5-8 cells appeared to have initiated soma growth. In addition, the extent of their growth was severely retarded (Fig. 6).

\section{Influence of juvenile hormone on IN cell fate}

For epidermal cells (Riddiford, 1980), motoneurons (Weeks and Truman, 1986), and sensory neurons (Levine et al., 1986), the small commitment peak of ecdysteroids serves to commit cells to pupal differentiation and renders them insensitive to the antimetamorphic effects of JH. For these tissues, treatment with $\mathrm{JH}$ or JHM on the second day of the 5 th instar (prior to the commitment peak) results in a larval response to endogenous or applied $20-\mathrm{HE}$, whereas the same treatment given to a wandering larva (after the commitment peak) leads to a pupal response. To determine if the same was true for the IN cells, we compared the response to treatment with JHM of abdomens isolated on the 2 nd day of the 5 th instar and from wandering animals (Table 1). On the day following treatment, subsets of the abdomens from both groups were infused with $20-\mathrm{HE}$.

JHM treatment alone had no overt effects on the fate of the IN cells in abdomens isolated either before or after the commitment peak. No signs of cell death or soma growth were seen in any of the nests, a condition identical to that seen in ligated control abdomens. As expected, treatment of abdomens isolated before the commitment peak with JHM blocked the metamorphic effects of $20-\mathrm{HE}$ infusion on the IN cells. There were no signs of either IN cell death or growth in abdomens treated with both hormones.

The response of the nests in abdomens isolated on the day of wandering, after they have been subjected to the small commitment peak of ecdysteroids, to application of both JHM and 20-HE was more complex. In M(A4), we observed a few pycnotic cells within the nest within $24 \mathrm{hr}$ after 20 -HE infusion in the presence of JHM. However, $5 \mathrm{~d}$ after infusion with 20-HE, the M(A4) nest showed no net loss of cells when compared with ligated controls. This suggests that the continuing activity of the $\mathrm{M}$ neuroblasts was able to compensate for the loss of cells trig- 
gered by the infusion of 20-HE. The major wave of ecdysoneinduced cell death in the nest was blocked by prior application of JHM. In N-T(A4), JHM treatment after the onset of wandering rescued approximately half the IN cells from $20-\mathrm{HE}$ triggered degeneration. In this nest, cell counts were reduced by only $25-35 \%$ (to 15 , rather than the expected $5-8$, cells).

The growth of the cells within the M(A4) nest was inhibited by treatment with JHM prior to infusion of 20-HE (Fig. 6). This effect was seen irrespective of whether the isolated abdomens had been exposed to the commitment pulse. Not only did fewer cells initiate growth, but the extent of growth was much less than in abdomens treated with 20-HE alone.

\section{Discussion}

Hormones serve as cues in the regulation of neural development in a number of systems. During amphibian metamorphosis, thyroxine has been implicated in the death of the Mauthner neuron (Kimmel and Model, 1978), as well as in the development of the ipsilateral retinothalamic projection (Hoskins and Grobstein, 1985). The vertebrate sex steroids are involved in regulating the events that sculpt the sexually dimorphic nuclei found within the CNS of a number of vertebrates (DeVoogd and Nottebohm, 1981; Gurney, 1981; Breedlove and Arnold, 1983). In moths, the ecdysteroids and the JHs play a major role in regulating the death or respecification of mature larval neurons during adult development (Truman, 1984; Truman and Schwartz, 1984; Bennett and Truman, 1985; Weeks and Truman, 1985, 1986; Levine et al., 1986). The present paper shows that these same hormones also regulate the fate of the progeny of postembryonic neuroblasts early in lepidopteran metamorphosis.

The sources of the ecdysteroids and JHs are in the thorax and head, respectively. Isolation of abdomens prior to the release of the small commitment peak of ecdysteroids prevented the resumption of IN cell development. No cell death or enlargement was observed within the nests. In sections of ganglia from larvae sacrificed after the release of the small commitment peak, there were a few degenerating IN cells within all of the A4 nests. In the $M(A 4)$ nest, there were also a few cells that had initiated some expansion. In abdomens isolated at this point, no further IN cell development occurred. Thus, the small commitment peak of ecdysteroids appears to have only a minor role in the fate of the IN cells. The major waves of cell loss and growth within the nests were correlated with the appearance of the large prepupal peak of ecdysteroids. The extent of the development observed among the IN cells depended on the proportion of the prepupal peak experienced by the abdomen prior to ligation. However, even when abdomens were isolated on the day of pupation, after the ganglia had experienced the full extent of the prepupal ecdysteroid peak, the number of cells seen in the nests was not equivalent to that observed in intact controls 2-3 d after pupation. Each of the A4 nests in such abdomens contained from 5 to 10 supernumerary cells. In addition, the extent of subsequent soma growth exhibited by individual IN cells was less than that observed in unligated controls. These results suggest there is a third period, occurring after pupation, that influences the fate of the IN cells within the abdominal ganglia. However, due to the difficulty in manipulating early pupae, no direct evidence for this third signal was collected.

When abdomens isolated on the day of wandering were given an infusion of 20-HE designed to mimic the prepupal peak, it led to a resumption of IN cell development within all the ab- dominal nests. Within $24 \mathrm{hr}$ of the start of the infusion both IN cell death and growth were observed in all the nests. The temporal order of the events observed in intact controls was maintained in the infused abdomens, although the infusion paradigm accelerated the process of cell loss. The number of cells surviving after 20-HE infusion in all the ncsts was greater than that seen in intact controls 3-4 d after pupation. In addition, the extent of cell growth observed was less than that seen in controls at this time. Both the extent of cell loss and soma growth were within the ranges observed in abdomens ligated on the day of pupation. In intact larvae the rise in the prepupal titer of ecdysteroids is accompanied, after a short delay, by a rise in the titer in JHs. However, in the isolated abdomens replacement of the ecdysteroids alone was sufficient to mimic completely the developmental events observed among the IN cells prior to pupation, but it does not carry their development beyond this point.

Although the commitment peak of ecdysteroids has only a minor effect on IN death or growth, it appears to increase the sensitivity of the IN cells to subsequent pulses of ecdysteroids. A $12 \mathrm{hr}$ infusion of 20 -HE into abdomens isolated before the commitment pulse triggered the resumption of IN cell development, but the extent of cell loss was only about $60 \%$ of that seen in 20-HE infused abdomens isolated from wandering animals. Similarly, in the M(A4) nests of infused abdomens isolated from prewandering larvae, both the number of IN cells exhibiting soma growth and the extent of their growth was substantially reduced compared to the same nest in 20-HE-treated abdomens isolated from wandering animals.

One surprising result was the persistence of JHM sensitivity of the IN cells after the onset of wandering. Unlike other larval tissues (Riddiford, 1980; Levine et al., 1986; Weeks and Truman, 1986), most of the IN cells do not commit to a pupal developmental program in response to the first small peak of ecdysteroids. The response of the IN cells to JHM treatment after wandering appeared to be lineage dependent. In abdomens isolated before the commitment peak, JHM application blocked the response of all the nests to a subsequent large pulse of ecdysteroids. When the same manipulations were performed on abdomens isolated from wandering larvae, the resumption of IN cell development was still effectively blocked in 3 of the 4 A4 nests. While a few degenerating cells appeared within these nests by $24 \mathrm{hr}$ after infusion of 20-HE, no net loss of cells was evident (suggesting that the small loss was offset by some residual IN cell birth). Of the 4 nests in A4, only the N-T nest showed a net loss of cells. However, even in this nest the extent of cell death after JHM treatment was only half of that seen in isolated abdomens treated with $20-\mathrm{HE}$ alone. In the M(A4) nest, the number of cells exhibiting ecdysteroid-induced growth was dramatically reduced by JHM treatment, and the extent of this growth was also retarded. It appears that, at most, only a small subset of the IN cells become pupally committed in response to the small peak of ecdysteroids. What accounts for this differential sensitivity to the commitment peak among the IN cells within a nest is not known.

Thus far, the IN cells are the only cells within the CNS of Manduca whose metamorphosis can still be inhibited by JHs following the commitment peak of ecdysteroids. In the case of motorneurons that degenerate at the larval-pupal transition, application of a $\mathrm{JH}$ analog to abdomens isolated after the onset of wandering does not affect their response to a subsequent infusion of 20-HE (Weeks and Truman, 1986). In intact larvae, 
the rising phase of the prepupal peak of ecdysteroids precedes the rise in JH titer by several hours (Riddiford, 1980). This interval may be sufficient to prevent the antimetamorphic effects of JHs on the subsequent development of the IN cells, in effect providing the uncommitted cells a second chance to become committed. Also, it has been reported that it is the inactive $\mathbf{J H}$ acid that is released at the time of the prepupal peak of ecdysteroids. Only those cells containing the JH methyltransferase can convert the $\mathrm{JH}$ acid to the active $\mathrm{JH}$ methyl ester (Sparagana et al., 1985). If the IN cells do not contain this enzyme, they would be nonresponsive to the surge of $\mathrm{JH}$ acid that is released at this time.

As the comparison of the results obtained from the $M$ and $\mathrm{N}-\mathrm{T}(\mathrm{A} 4)$ nests makes clear, the response of the IN cells in both intact animals and isolated abdomens treated with JHM and 20-HE was lineage dependent, with each nest playing out its own developmental program in response to the appropriate hormonal cues. The JHs and ecdysteroids appear to serve only as developmental signals, not as determinants of cell fate.

These results suggest that ecdysteroids and JHs play a central role in controlling the developmental fate of the IN cells. However, they do not address whether the IN cells are themselves the targets or if they are indirectly affected. There is a possibility that other cells are actually the targets of these hormones, and it is the response of these cells that indirectly serves as the trigger for the resumption of IN cell development. This scenario seems unlikely in light of the JHM results. Application of JHM to animals after the start of wandering has no effect on preexisting larval motorneurons or sensory neurons. The retraction of dendrites and subsequent death of motoneurons (Weeks and Truman, 1985, 1986), as well as the respecification of sensory neurons, occurs on schedule (Levine et al., 1986). Yet, the development of most of the IN cells remained arrested. Consequently, the response of these cells appears to be independent of events that occur in the rest of the CNS.

\section{References}

Abercrombie, M. (1946) Estimation of nuclear population from microtome sections. Anat. Rec. 94: 239-247.

Bell, R. A., and F. A. Joachim (1978) Techniques for rearing laboratory colonies of the tobacco hornworm and pink bollworms. Ann. Ent. Soc. Am. 69: 365-373.

Bennett, K. L., and J. W. Truman (1985) Steroid-dependent survival of identifiable neurons in cultured ganglia of the moth Manduca sexta. Science 229: 58-60.

Booker, R., and J. W. Truman (1987) Postembryonic neurogenesis in the CNS of the tobacco hornworm, Manduca sexta. I. Neuroblast arrays and the fate of their progeny during metamorphosis. J. Comp. Neurol. 255: 548-559.

Breedlove, S. M., and A. P. Arnold (1983) Hormonal control of a developing neuromuscular system. II. Sensitive periods for the androgen-induced masculinization of the rat spinal nucleus of the bulbocavernosus. J. Neurosci. 3: 424-432.

DeVoogd, T. J., and F. Nottebohm (1981) Gonadal hormones induce dendritic growth in the adult avian brain. Science 214: 202-204.

Ephrussi, B., and G. W. Beadle (1936) A technique of transplantation for Drosophila. Am. Nature 70: 218-225.

Gurney, M. (1981) Hormonal control of cell form and number in the zebra finch song system. Brain Res. 231: 153-172.

Hoskins, S. G., and P. Grobstein (1985) Development of the ipsilateral retinothalamic projection in the frog Xenopus laevis. III. The role of thryoxine. J. Neurosci. 5: 930-940.

Kiguchi, K., and L. M. Riddiford (1978) The role of juvenile hormone in pupal development of the tobacco hornworm, Manduca sexta. $\mathrm{J}$. Insect Physiol. 24: 673-680.

Kimmel, C. B., and P. Model (1978) Developmental studies of the Mauthner cell. In Neurobiology of the Mauthner Cell, D. S. Faber and H. Horn, eds., pp. 183-220, Raven, New York.

Levine, R. B., and J. W. Truman (1982) Metamorphosis of the insect nervous system: Changes in the morphology and synaptic interactions of identified neurons. Nature 299: 250-252.

Levine, R. B., and J. W. Truman (1985) Dendritic reorganization of of abdominal motorneurons during metamorphosis of the moth, Manduca sexta. J. Neurosci. 5: 2424-2431.

Levine, R. B., J. W. Truman, D. Linn, and C. M. Bate (1986) Endocrine regulation of the form and function of axonal arbors during insect metamorphosis. J. Neurosci. 6: 293-299.

Meltzer, Y. L. (1971) Hormonal and Attractant Pesticide Technology. Noyes Data Corp., Park Ridge, N. J.

Nijhout, H. F. (1976) The role of ecdysone in pupation of Manduca sexta. J. Insect. Physiol. 22: 453-463.

Richardson, K. C., L. Jarett, and E. H. Finke (1960) Embedding in epoxy resins for ultrathin sectioning in electron microscopy. Stain Technol. 35: 313-323.

Riddiford, L. M. (1980) Interaction of ecdysteriods and juvenile hormone in the regulation of larval growth and metamorphosis of the tobacco hornworm. In Progress in Ecdysone Research, J. A. Hoffman, ed., pp. 409-430, Elsevier/North Holland Biomedical, Amsterdam.

Sparagana, S. P., G. Bhaskaran, and P. Barrera (1985) Juvenile hormone acid methyltransferase activity in the imaginal discs of $\mathrm{Man}$ duca sexta prepupae. Arch. Insect Biochem. Physiol. 2: 191-202.

Taylor, H. M., and J. W. Truman (1974) Metamorphosis of the abdominal ganglia of the tobacco hornworm, Manduca sexta: Changes in the population of identified motor neurons. J. Comp. Physiol. 90: 367-388.

Truman, J. W. (1984) Cell death in invertebrate nervous systems. Ann. Rev. Neurosci. 7: 171-188.

Truman, J. W., and S. E. Reiss (1976) Dendritic reorganization of an identified motoneuron during metamorphosis of the tobacco hornworm moth. Science 192: 477-479.

Truman, J. W., and L. M. Schwartz (1984) Steroid regulation of neuronal death in the moth nervous system. J. Neurosci. 4: 274-280.

Weeks, J. C., and J. W. Truman (1985) Independent steroid control of the fate of motoneurons and their muscles during insect metamorphosis. J. Neurosci. 5: 2290-2300.

Weeks, J. C., and J. W. Truman (1986) Hormonally mediated reprogramming of muscles and motoneurones during the larval-pupal transformation of the tobacco hornworm, Manduca sexta. J. Exp. Biol. 125: 1-13. 